\title{
Effects of Education, Vaccination and Treatment on HIV Transmission in Homosexuals with Genetic Heterogeneity
}

\author{
Cornell Univ., Dept. of Biometrics Technical Report BU-1587-M
}

\author{
Sara Del Valle \\ University of Iowa, Iowa City, IA
}

Arlene Morales Evangelista

Arizona State University, Tempe, AZ

Maria Cristina Velasco

Universidad del Valle, Cali,Colombia

August 2001

\begin{abstract}
Genetic studies report the existence of a mutant allele $\Delta 32$ of $C C R 5$ chemokine receptor gene at high allele frequencies $(\sim 10 \%)$ in Caucasian populations. The presence of this allele is believed to provide partial or full resistance to HIV. In this study, we look at the impact of education, temporarily effective vaccines and therapies on the dynamics of HIV in homosexually active populations. In our model, it is assumed that some individuals possess an allele (like $\Delta 32$ of CCR5) that prevents the successful invasion or replication of HIV. Our model therefore differentiates by genetic and epidemiological status and naturally ignores the reproduction process. Furthermore, HIV infected individuals are classified as rapid, normal or slow progressors. In this complex setting, the basic reproductive number $\Re_{0}$ is derived in various situations. The separate or combined effect of therapies, education and vaccines are analyzed. Our results support the conclusions of Shu-Fang Hsu Schmitz that some integrated intervention strategies are far superior to those based on a single approach.
\end{abstract}




\section{Background}

The human immunodeficiency viruses (HIV-1 and HIV-2), are pathogens that causes the deadly disease of the acquired immune deficiency syndrome (AIDS). The origins of these viruses, HIV-1 and HIV-2 are not known, but the first known cases in the U.S were reported in gay men from San Francisco in 1981. Even though HIV is considered to be a relatively young disease, evidence has shown a case of HIV-1 positive from Zaire in 1959. Some evolutionary biologist belief that the virus was first transmitted to humans from monkey viruses. Humans, transmitted the disease mainly through sexual intercourse and intravenous drug users. During the 1980's and 1990's two waves of HIV infection emerged: one among heterosexual men and women in Africa, and two among homosexual men and intravenous drug users in the U.S, as well as in Europe. The rates of HIV infection in Africa are very high compared to U.S and Europe. Currently, AIDS is the fourth leading cause of death globally and the leading cause in Africa. For the year 1996, approximately 23 million people were HIV positive world wise. As of today, 36 million people have been reported to be HIV positive worldwide [14]. These shows a difference of approximately 13 million new infecteds, which shows the rapid transmission of HIV. [14]

HIV specifically targets the "white blood" cells, i.e helper T cells. Helper T cells are vital for the function of the immune system since their job is to activate killer $\mathrm{T}$ cells to destroy anything that is foreign to the human body. HIV attaches itself to the CD4 protein (a receptor) from the helper T cells, and once there it uses the host cell's enzymatic machinery to reproduce, and grow. Once the HIV is inside the host cell, the virus uses reverse transcriptase to copy its RNA genome into double stranded DNA molecules. This double helix enters the host cell's nucleus, where the virus genome integrates into the host chromosome. In most cases, this infected chromosome replicates two new infective cells for each cell division. The immune system responds to HIV infection by destroying the virions floating on the bloodstream, or by killing its own infected $\mathrm{T}$ cells. The life cycle and gene composition of HIV-2 is very similar to that of HIV-1. Yet HIV-2 is much more benign and has a much lower mutation rate than HIV-1's reverse transcriptase. [21] In this research we are only working with the human immunodeficiency virus-1.

HIV does not kill people directly or quickly; instead, the destruction of CD4+ $\mathrm{T}$ cells weakens the immune system. Reverse transcription is high error prone and $\mathrm{T}$ cells are not able to recognize all of the altered epitotes in order to destroy them. This is because the immune system is limited to recognize certain proteins or epitotes as foreign. HIV is constantly changing its epitotes because the virus uses reverse transcription for its reproduction. Consequently, the immune system is not able to keep up with all the mutations, and the virions overturn the $\mathrm{T}$ cells, in the long run the immune system collapses. This is the last of a three-phase sequence of AIDS. In the first phase, $30 \%$ of those infected, the person presents symptoms of skin rashes and fever, but this only lasts a few weeks. The duration of the second phase has an average of ten years. In this phase, T cells and HIV are constantly fighting for survival, but as time progresses the number of $\mathrm{T}$ cells continues to decline from a "normal level of about 1,000 cells/milliliter of blood to only 200 cells/mL." It is at this threshold that infected individuals enter the third AIDS phase. The disintegration of the immune system happens because it can no longer defend itself against other bacterial and fungal pathogens, especially when most $\mathrm{T}$ cells are dead or 
infected by the virus. The expected life for individuals in this phase is about 2 years. [21]

The devasting global impact of HIV has increased research efforts to find an effective vaccine, or drugs that would stop the progression and transmission rate of HIV. These efforts have been mildly successful since HIV evolves resistance to drugs and mutates extremely fast. Recent genetics studies indicate a correlation of mutant genes in HIV co-receptors that may provide protection against HIV. This gives us the hope that researchers may be able to develop a drug that may mimic the resistant gene or that gene therapy may be useful in preventing HIV. This "insertion' of a resistant gene might be able to stop the HIV infection from progressing to AIDS, and therefore lowering the transmission rates of HIV/AIDS. 


\section{Introduction}

Recent genetic studies observed that many individuals with multiple exposure to HIV-1 remain seronegative, while others continue to be infected by HIV-1. Researchers have correlated these findings with some mutant genes in HIV co-receptors. Studies found the presence of mutant alleles such as $\Delta 32$ and $\mathrm{m} 303$ of CCR5 suggesting resistance or protection against HIV for some individuals. Our interest lies precisely on these mutant alleles and the receptors from the host's cell and the virus's cell. It seems that mutant alleles have somehow change the structure of the helper $\mathrm{T}$ cells in a way that it is very difficult for the virus' receptor to connect to it. Hence, the virus stops replicating and individuals show a resistant to HIV. According to the findings, the mutant alleles seem to provide full protection against HIV in homozygotes, and partial resistance for heterozygotes. These results were observed in about $10 \%$ of the Caucasian population. For Hsu Schmitz [2] these conclusions indicated the "existence of genetic heterogeneity with respect to susceptibility to HIV infection and to rate of AIDS progression in general populations". Therefore, using this inference Hsu Schmitz investigated the impact of genetic heterogeneity via a deterministic model for homosexually active population. Hsu Schmitz concluded that giving treatment and vaccination to patients was helpful in reducing the transmission rate, but they were not helpful if they were implemented alone. This project starts from Hsu Schmitz's basic ideas and model. A similar model is constructed in which a susceptible population is subject to vaccination, education and treatment. Our primary goal is to investigate the effects of education, temporary vaccination and treatment on HIV transmission in a homosexually active population with genetic heterogeneity.

Vaccination and treatment are not efficient control methods. Vaccines in development may only give a temporary immunity to HIV/AIDS. Antiviral drugs used in treatment such as AZT (zidovudine), ddc(dideoxycytidine), and ddl(dideosyinosine) also worked temporarily. Theses antiviral drugs blocks the replication of the virus. However, the virus's high rate of mutation eventually catches on. Those two methods are costly both at the individual and population level. Hence, education may be the key factor in altering the course of this detrimental world pandemic. In our model, education means that individuals are encouraged to use condoms, or have fewer partners. Abstinence and safe sexual behavior are also involved. This paper is divided into the following sections. Section 2 explains the complex model and includes the diagram of the compartmental model which illustrates the dynamics of the population under study. Section 3 explains in great detail the different cases for the reproductive number, $R_{0}$. Conclusions and final thoughts are in Section 4. Acknowledgments are given in Section 5. 


\section{The Complex Model}

Following Hsu Schmitz [1] we classify the homosexually active population into three classes of susceptible individuals: no resistant $\left(S_{1}\right)$, partially resistant $\left(S_{2}\right)$ and fully resistant $\left(S_{3}\right)$ to HIV infection. Infected individuals are classified as rapid $\left(I_{1}\right)$, normal $\left(I_{2}\right)$ and slow $\left(I_{3}\right)$ progressors. Throughout this paper, the index $i$ refers to the non infected groups, i.e.susceptible, vaccinated and educated individuals and the index $j$ refers to infected classes capable of transmitting the disease, i.e.,infected and treated individuals. In this model we assume that AIDS patient are sexually inactive, hence, AIDS patients do not affect the HIV process.

As in Hsu Schmitz (1999), we assume that recruitment occurs at a constant rate, $\pi$. The three susceptible groups get the respective fixed fractions, $g_{i}\left(i=1,2,3\right.$ and $\left.\sum_{i} g_{i}=1\right)$, which are related to the frequencies of relevant genotypes. Although genotype frequencies usually change with time due to random fluctuation and/or to disease, the frequencies in a homosexually population will have little effect on new recruits, hence we ignore them. Because in general the frequencies of mutant alleles are relatively small, it is expected and reseanable to assume that

$$
g_{1}>g_{2}>g_{3}
$$

that is, most individuals have no resistance, a small fraction have partial resistance, and an even smaller fraction have complete resistance. All individuals are subject to the common per-capita natural removal rate, $\mu$.

The average number of partners per unit time is denoted by $c_{i}(i=1,2,3)$ for $S_{i}$-individuals and for $I_{j}$-individuals. As in Hsu Schmitz's paper we assume that genetic heterogeneity does not influence pairing behavior because most individuals do not know their genotypes at loci related to HIV susceptibility and/or AIDS pathogenesis. Furthermore, we assume that all individuals of a given disease status have the same average number of partners per unit time, i.e., $c_{i}=c$ for all $i$. To make the model simpler, as Hsu Schmitz we further assume that disease status does not affect pairing behavior, as in Anderson, Gupta and May (1991), McLean and Blower (1993), and Castillo-Chavez, et, al (1989a, b). We follow this same approach.

The per-capita progression rates for $I_{j}$ individuals are denoted by $\gamma_{j}(j=1,2,3)$. Because $1 / \gamma_{j}$ is the average incubation time of $I_{j}$-individuals, it is obvious that

$$
\gamma_{1}>\gamma_{2}>\gamma_{3}
$$

The infectiousness of $I_{j}$ individuals is reflected by the per-partnership transmission rate, $\beta_{j}(j=1,2,3)$. We assume that fast progressors $\left(I_{1}\right)$ have the highest viral load, thus are most infectious. In addition, slow progressors $\left(I_{3}\right)$ have the lowest viral load, hence are least infectious. More specifically, we hypothesize the following relation:

$$
\beta_{1} \geq \beta_{2} \geq \beta_{3}
$$

It has been shown that the viral load and the infectiousness may change dramatically during the incubation period. However, to incorporate this fact we would need to keep track of the 
"age" of infection of each individual and end up with a complicated model [22]. For the sake of simplicity, here we assume $\beta_{j}$ are constant as in Anderson, Gupta and May (1991), in McLean and Blower (1993) and Castillo-Chavez, et, al (1989a, b).

During the partnership between an $S_{2}$-individual and an $I_{j}$-individual $(\mathrm{j}=2,3)$, the transmission rate $\beta_{j}$ of the infected partner is reduced to $x_{j} \beta_{j}$, with $0<x_{j}<1$ to account for partial resistance to HIV in $S_{2}$-individuals. Newly infected $S_{i}$-individuals $(i=1,2)$ join the three infected groups with respective proportions $f_{i j}$, which satisfy:

$$
0 \leq f_{i j} \leq 1 \text { and } \sum_{j=1}^{3} f_{i j}=1 .
$$

We expect the new infecteds who have no resistance $\left(S_{1}\right)$ to generate a larger fraction of rapid progressors $\left(I_{1}\right)$ and a smaller fraction of slow $\left(I_{3}\right)$ progressors than those coming from $S_{2}$, that is,

$$
f_{11}<f_{21} \text { and } f_{13}<f_{23} .
$$

A certain number of individuals from the susceptible class $S_{i}(i=1,2,3)$ are assumed to be educated at a rate $\alpha$. A certain proportion $\left(p_{i}\right)$ of newly recruited $S_{i}$-individuals is assumed to be effectively vaccinated. Moreover a certain number of individuals from the infected $I_{j^{-}}$ individuals $(j=1,2,3)$ are assumed to be effectively treated at a rate, $\left(m_{j}\right)$. We assume that the transmission rate of effectively treated individuals $\left(T_{j}\right)$ is reduced from $\beta_{j}$ to $a \beta_{j}$ with $0 \leq a<1$, and the progression rate is reduced from $\gamma_{j}$ to $y \gamma_{j}$ with $0 \leq y<1$. The value of 1 is not included in the ranges of $a$ and $y$ because it means treatment does not reduce infectiousness and rate of progression at all, which does not sound reasonable based on current knowledge. As in [10], and [2] we assume that the vaccines have a "take" proportion of $\epsilon(0<\epsilon<1)$, an efficacy of $\xi(0<\xi<1)$ and a protection duration of $1 / \omega$ units of time. The ranges of $\epsilon$ and $\xi$ do not include 0 and 1 because 0 implies the vaccine is useless and 1 implies the vaccine is perfect; both are unrealistic. These vaccine parameters indicate $[100 \times(1-\epsilon)] \%$ vaccinated individuals are not effectively vaccinated and they are in principle like other unvaccinated individuals. As in [2], those effectively vaccinated individuals (in a proportion of $p_{i} \epsilon$ ), denoted by $V_{i}$, still have $[100 \times(1-\xi)] \%$ chance to be infected within the protection duration of $1 / \omega$ units of time. No reduction in infectiousness is assumed for infecteds who have been vaccinated. Although individuals might become more active (i.e., having more sexual partners per unit of time) after being treated or vaccinated, for simplicity we assume as in [2] that neither the treatment nor the vaccination changes people's pairing behavior, so the common pairing activity $c$ and the proportional mixing pattern are still in effect. In addition, we assume that the effectiveness of education is given by $\Psi$ and its range does not include 0 and 1 because 0 implies that education is useless and 1 implies that education is completely effective. Now, let the total population be denoted by

$$
\Phi:=\sum_{i=1}^{3}\left(V_{i}+S_{i}+E_{i}\right)+\sum_{j=1}^{3}\left(I_{j}+T_{j}\right)
$$


We reparameterize the transmission rates via $\beta:=\beta_{2}$, or more specifically,

$$
\beta_{1}=b_{1} \beta \text { and } \beta_{3}=b_{3} \beta .
$$

Relation (3) implies that the multipliers

$$
b_{1} \geq 1 \text { and } 0 \leq b_{3} \leq 1
$$

Currently there are no data that throw light on whether or not the reduction factors $x_{j}$ for $\beta_{j}$ depend on $j$. To continue our goal of analyzing the simplest possible genetic-epidemiological model, we assume that $x_{j}=x$ for all $j$.

Then the forces of infection for $S_{1}$ and $S_{2}$ individuals are

$$
\begin{aligned}
\sigma_{S_{1}} & =\beta\left(\sum_{j=1}^{3} b_{j} I_{j}+a \sum_{j=1}^{3} b_{j} T_{j}\right) / \Phi \\
\sigma_{S_{2}} & =x \sigma_{S_{1}}
\end{aligned}
$$

for $E_{1}$ - and $E_{2}$-individuals are

$$
\begin{aligned}
\sigma_{E_{1}} & =(1-\Psi) \sigma_{S_{1}} \\
\sigma_{E_{2}} & =x \sigma_{V_{1}} \\
& =x(1-\Psi) \sigma_{S_{1}}
\end{aligned}
$$

and for $V_{1}$ - and $V_{2}$-individuals are

$$
\begin{aligned}
\sigma_{V_{1}} & =(1-\xi) \sigma_{S_{1}} \\
\sigma_{V_{2}} & =x \sigma_{V_{1}} \\
& =x(1-\xi) \sigma_{S_{1}}
\end{aligned}
$$

The numbers of newly infected $S_{i^{-}}, V_{i^{-}}$and $E_{i^{-}}$individuals $(i=1,2)$ are

$$
\begin{aligned}
\delta_{S_{i}} & =c S_{i} \sigma_{S_{i}}, \\
\delta_{V_{i}} & =c V_{i} \sigma_{V_{i}}, \\
\delta_{E_{i}} & =c E_{i} \sigma_{E_{i}} .
\end{aligned}
$$

These newly infected individuals enter the $j$ th $(j=1,2,3)$ infected group $\left(I_{j}\right.$ and $\left.T_{j}\right)$ at the rate

$$
\rho_{j}=\sum_{i=1}^{2} f_{i j}\left(\delta_{S_{i}}+\delta_{V_{i}}+\delta_{E_{i}}\right) .
$$




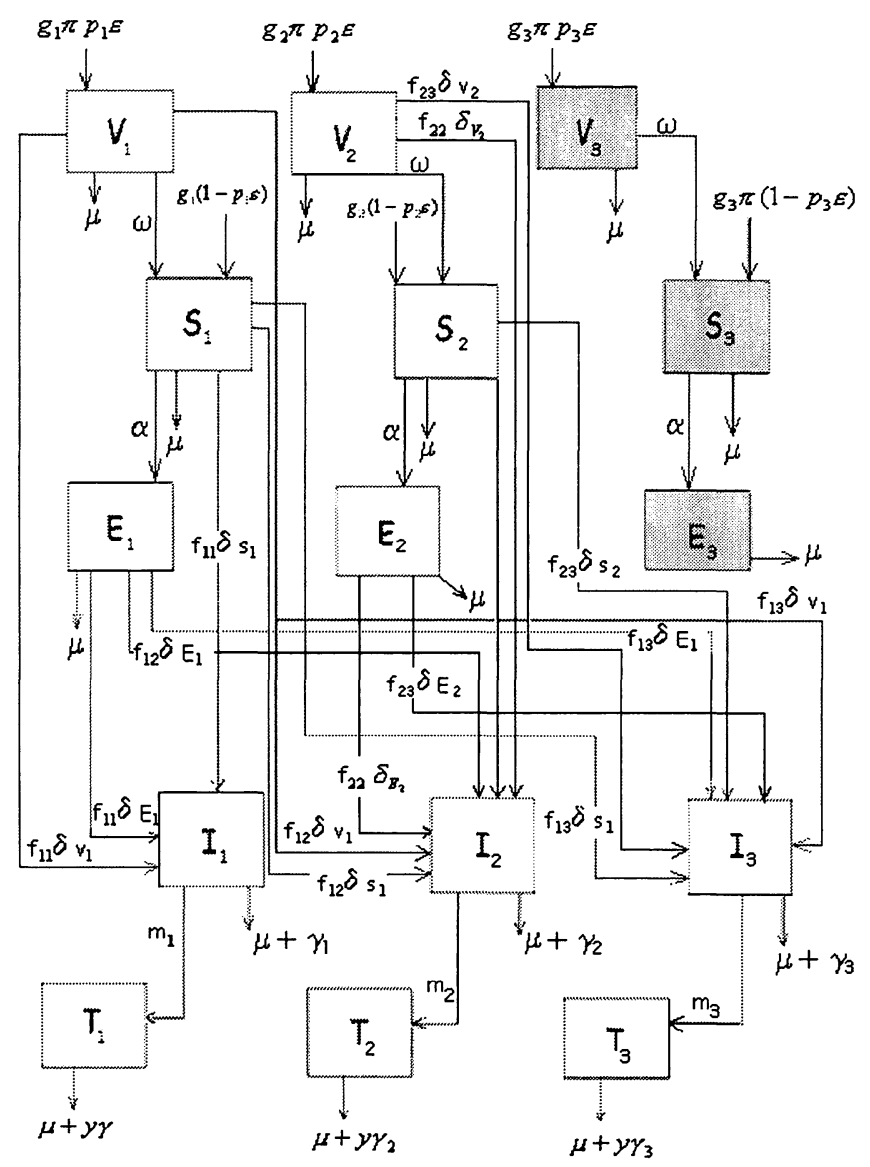

Figure 1: Diagram of the Compartmental Model. 
The mathematical model is described by the following system of equations, where $i=1,2$ and $\mathrm{j}=1,2,3$ :

$$
\begin{aligned}
\dot{V}_{i} & =g_{i} \pi p_{i} \epsilon-(\mu+\omega) V_{i}-\delta_{V_{i}} \\
\dot{V}_{3} & =g_{3} \pi p_{3} \epsilon-(\mu+\omega) V_{3} \\
\dot{S}_{i} & =g_{i} \pi\left(1-p_{i} \epsilon\right)+\omega V_{i}-(\mu+\alpha) S_{i}-\delta_{S_{i}} \\
\dot{S}_{3} & =g_{3} \pi\left(1-p_{3} \epsilon\right)+\omega S_{3}-(\mu+\alpha) S_{3} \\
\dot{E}_{i} & =\alpha S_{i}-\mu E_{i}-\delta_{E_{i}} \\
\dot{E}_{3} & =\alpha S_{3}-\mu E_{3} \\
\dot{I}_{j} & =\rho_{j}-\left(\mu+m_{j}+\gamma_{j}\right) I_{j} \\
\dot{T}_{j} & =m_{j} I_{j}-\left(\mu+y \gamma_{j}\right) T_{j} \\
P(t) & =\sum_{k=1}^{3}\left(V_{k}+S_{k}+E_{k}+I_{k}+T_{k}\right)
\end{aligned}
$$

\subsection{Simple model}

For interpretation purposes we first considered a homozygotic population (note that the frequencies of genotypes is equal to 1). By doing so we obtained the following reproductive number (where the subscript S denotes "Simple")

$$
\Re_{0_{S}}=\frac{1}{2}\left\{\frac{f c \beta b k}{\mu+m+\gamma}+\sqrt{\left(\frac{f c b k \beta}{\mu+m+\gamma}\right)^{2}+4 a\left(\frac{f b k c m \beta}{(\mu+y \gamma)(\mu+m+\gamma)}\right)}\right\}
$$

where

$$
k=\left(1-\frac{\alpha}{\mu+\alpha} \Psi\right)\left(1-\frac{\mu}{\mu+\omega} p \epsilon\right)+(1-\xi)\left(\frac{\mu}{\mu+\omega}\right)
$$

It is worthwhile to notice that $\mathrm{k}$ contains only parameters involving education and vaccination. The first two terms denote the reduction factor for education and the proportion of non-vaccinated individuals respectively. The other two terms represent the reduction factor for vaccination and the proportion of vaccinated individuals respectively. Notice that the range of $\mathrm{k}$ will always be between 0 and 1 , which implies that education and vaccination will always reduce the value of $\Re_{0_{S}}$.

For convenience let

$$
\Re_{0_{S I}}=\frac{f c \beta b k}{\mu+m+\gamma} \quad \text { and } \quad \Re_{0_{S T}}=\frac{m}{\mu+y \gamma}
$$

by making these substitutions we can write $\Re_{0_{S}}$ as follows

$$
\Re_{0_{S}}=\frac{1}{2}\left(\Re_{0_{S I}}+\sqrt{\Re_{0_{S I}}\left(\Re_{0_{S I}}+4 a \Re_{0_{S T}}\right)}\right)
$$


The square root in $\Re_{0}$ represents the 2 step process that a treated person takes before $\mathrm{s} / \mathrm{he}$ can actually generate another treated individual. In order to analyze the value of $\Re_{0_{S}}$, we shall take its derivative with respect to $\mathrm{m}$ and show under what circumstances,

$$
\frac{d \Re_{0_{S T}}}{d m}<0
$$

which implies that increasing treatment lowers $\Re_{0_{S}}$. Thus by taking the derivative we obtain

$$
\frac{2 a \eta}{\eta_{y}}-\frac{f c b k \beta}{\mu+m+\gamma}-\sqrt{\left(\frac{f c b k \beta}{\mu+m+\gamma}\right)^{2}+\frac{4 a f b k c m \beta}{(\mu+y \gamma)(\mu+m+\gamma)}}<0
$$

which gives the following inequality

$$
a<\Re_{0_{S T}}(0)\left(\frac{\mu+y \gamma}{\mu+\gamma}\right)
$$

Hence, treatment will reduce $\Re_{0_{S T}}$. We should start a treatment program, only if it reduces the infectivity by at least this factor. Reducing infectivity is more beneficial than letting people live longer, since the more an individual lives the more people s/he infects, so the good effect of treatment (a) has to outweigh the "bad" effect (y) on the population. Hence, by increasing treatment we will be able to lower the value of $\Re_{0}$. The analysis of this homozygotic population would be helpful to understand the analysis of $\Re_{0}$ for the heterogeneity population, which is presented in the next section.

\section{The Basic Reproductive Number}

\subsection{The $\Re_{0}$ of the Complex Model}

As mentioned in [7] the reproductive number $\left(\Re_{0}\right)$ is the effected number of secondary cases produced by a typical infected individual during its entire period of infectiousness in a demographically steady susceptible population. Therefore, in order to study whether HIV will invade a population or stabilize over a given region we must investigate the dynamics of $\Re_{0}$.

We are now ready to move on and compute the reproductive number when treatment, education and vaccination are applied to the population. To study the potential of disease spreading, we shall compute the basic reproductive number, $R_{0}$ as in Diekmann et al.( 1990), and Castillo-Chavez et al (forthcoming), which indicates whether disease may invade a population in demographic steady state when there is no disease present. The computation is done by linearizing our system (20) around the disease-free state and looking for conditions that guarantee the growth of the three infected classes, $I_{j}$ as well as the three treated classes, $T_{j}$.

The resulting 6-dimensional system is represented in the following form: 


$$
\dot{\mathbf{X}}=(\mathbf{M}-\mathbf{D}) \mathbf{X}
$$

where

$$
\begin{aligned}
& \mathbf{X}=\left[\begin{array}{c}
I_{1} \\
I_{2} \\
I_{3} \\
T_{1} \\
T_{2} \\
T_{3}
\end{array}\right], \quad \mathbf{D}=\left[\begin{array}{cccccc}
\eta_{1} & 0 & 0 & 0 & 0 & 0 \\
0 & \eta_{2} & 0 & 0 & 0 & 0 \\
0 & 0 & \eta_{2} & 0 & 0 & 0 \\
0 & 0 & 0 & \varphi_{1} & 0 & 0 \\
0 & 0 & 0 & 0 & \varphi_{2} & 0 \\
0 & 0 & 0 & 0 & 0 & \varphi_{3}
\end{array}\right] \\
& \mathbf{M}=c \beta\left[\begin{array}{ccccccc}
b_{1} \tau_{1} & \tau_{1} & b_{3} \tau_{1} & a b_{1} \tau_{1} & a \tau_{1} & a b_{3} \tau_{1} \\
b_{1} \tau_{2} & \tau_{2} & b_{3} \tau_{2} & a b_{1} \tau_{2} & a \tau_{2} & a b_{3} \tau_{2} \\
b_{1} \tau_{3} & \tau_{3} & b_{3} \tau_{3} & a b_{1} \tau_{3} & a \tau_{3} & a b_{3} \tau_{3} \\
m_{1} & 0 & 0 & 0 & 0 & 0 \\
0 & m_{2} & 0 & 0 & 0 & 0 \\
0 & 0 & m_{3} & 0 & 0 & 0
\end{array}\right]
\end{aligned}
$$

with

$$
\begin{aligned}
\tau_{1} & =f_{11}\left(\delta_{S_{1}}+\delta_{E_{1}}+\delta_{V_{1}}\right) \\
\tau_{2} & =f_{12}\left(\delta_{S_{1}}+\delta_{E_{1}}+\delta_{V_{1}}\right)+x f_{22}\left(\delta_{S_{2}}+\delta_{E_{2}}+\delta_{V_{2}}\right), \\
\tau_{3} & =f_{13}\left(\delta_{S_{1}}+\delta_{E_{1}}+\delta_{V_{1}}\right)+x f_{23}\left(\delta_{S_{2}}+\delta_{E_{2}}+\delta_{V_{2}}\right), \\
\varphi_{i} & =\mu+y \gamma_{i} \\
\eta_{i} & =\mu+m_{i}+\gamma_{i}
\end{aligned}
$$

The six eigenvalues of the matrix $\mathrm{MD}^{-1}$ are $0,0,0,0, \lambda_{-}$and $\lambda_{+}$, which are given by

$$
\lambda_{-}=\frac{1}{2}\left(\Re_{0_{I}}-\sqrt{\Re_{0_{I}}^{2}+4 a \overrightarrow{\Re_{I}} \cdot \overrightarrow{\Re_{T}}}\right) \quad \text { and } \quad \lambda_{+}=\frac{1}{2}\left(\Re_{0_{I}}+\sqrt{\Re_{0_{I}}^{2}+4 a \vec{\Re}_{I} \cdot \Re_{T}}\right)
$$

where

$$
\begin{aligned}
\Re_{0_{I}} & =c\left[k_{1}\left(f_{11} \frac{\beta_{1}}{\eta_{1}}+f_{12} \frac{\beta_{2}}{\eta_{2}}+f_{13} \frac{\beta_{3}}{\eta_{3}}\right)+x k_{2}\left(f_{22} \frac{\beta_{2}}{\eta_{2}}+f_{23} \frac{\beta_{3}}{\eta_{3}}\right)\right] \\
\overrightarrow{\Re_{I}} & =\left(\frac{k_{1} f_{11} \beta_{1}}{\eta_{1}},\left(k_{1} f_{12}+x k_{2} f_{22}\right) \frac{\beta_{2}}{\eta_{2}},\left(k_{1} f_{13}+x k_{2} f_{23}\right) \frac{\beta_{3}}{\eta_{3}}\right) \\
\overrightarrow{\Re_{T}} & =\left(\frac{m_{1}}{\varphi_{1}}, \frac{m_{2}}{\varphi_{2}}, \frac{m_{3}}{\varphi_{3}}\right)
\end{aligned}
$$


and

$$
k_{i}=g_{i}\left[\left(1-\Psi \frac{\alpha}{\mu+\alpha}\right)\left(1-P_{i} \epsilon \frac{\mu}{\mu+\omega}\right)+(1-\xi)\left(P_{i} \epsilon \frac{\mu}{\mu+\omega}\right)\right]
$$

Because all elements on the right hand side of $\lambda_{+}$are positive, it is clear $\lambda_{+}>0$. Therefore, $\lambda_{+}$ is the dominant eigenvalue of $\mathrm{MD}^{-1}$, which is also referred to the basic reproductive number, $\Re_{0}$ (Diekmann et al. 1990). Further issues about $\Re_{0}<1$ are discussed later when different cases are studied. The reproductive number for the complex model, $\Re_{0}$ is a combination of the secondary infections caused by the infected and treated class. Notice that the square root denotes the 2 step process that a treated individual takes before $\mathrm{s} /$ he generates another treated person, (since when $\mathrm{s} /$ he infects a person the person must become infected first before $\mathrm{s} / \mathrm{he}$ gets treated).

\subsection{Global Stability of Disease Free Equilibrium}

Here we define a Lyapunov function to prove that disease free equilibrium (DFE) is globally stable in the absence of an endemic equilibrium.

Theorem 3.1 Suppose that

$$
c \beta \sum_{k=1}^{3}\left(g_{1} f_{1 k}+g_{2} f_{2 k}\right) \frac{b_{3}}{\mu+\gamma_{3}}<1
$$

and

$$
c \beta \sum_{k=1}^{3}\left(g_{1} f_{1 k}+g_{2} f_{2 k}\right) \frac{a b_{3}}{\mu+y \gamma_{3}}<1
$$

Then the disease-free equilibrium is globally stable. 
Proof Let $L=\sum_{j}\left(I_{i}+T_{j}\right)$ be a Lyapunov function.

$$
\begin{aligned}
L^{\prime}(t) & =\sum_{j=1}^{3}\left\{\left(\mu+y \gamma_{j}\right) T_{j}-\left(\mu+\gamma_{j}\right) I_{j}\right. \\
& +\frac{c \beta}{\Phi} \sum_{k=1}^{3} b_{k}\left(I_{k}+a T_{k}\right)\left[f_{1 j}\left(S_{1}+(1-\xi) V_{1}+(1-\Psi) E_{1}\right)+x f_{2 j}\left(S_{2}+(1-\xi) V_{2}+(1-\Psi) E_{2}\right)\right] \\
& <\sum_{j=1}^{3}\left\{c \beta \frac{\sum_{k=1}^{3} b_{k}\left(I_{k}+a T_{k}\right)}{\Phi}\left(g_{1} f_{1 j}+g_{2} f_{2 j}\right) \frac{\pi}{\mu}-\left(\mu+\gamma_{j}\right) I_{j}-\left(\mu+y \gamma_{j}\right) T_{j}\right\} \\
& <\left[c \beta \sum_{k=1}^{3}\left(g_{1} f_{k 1}+x g_{2} f_{2 k}\right) \sum_{j=1}^{3} b_{j} I_{j}-\sum_{j}\left(\mu+\gamma_{j}\right) I_{j}\right] \\
& +\left[a c \beta \sum_{k=1}^{3}\left(g_{1} f_{k 1}+x g_{2} f_{2 k}\right) \sum_{j=1}^{3} b_{j} T_{j}-\sum_{j}\left(\mu+y \gamma_{j}\right) T_{j}\right] \\
& =\sum_{j=1}^{3}\left(\mu+\gamma_{j}\right)\left[c \beta \sum_{k=1}^{3}\left(g_{1} f_{1 k}+x g_{2} f_{2 k}\right) \frac{b_{j}}{\mu+\gamma_{j}}-1\right] I_{j} \\
& +\sum_{j=1}^{3}\left(\mu+y \gamma_{j}\right)\left[c \beta \sum_{k=1}^{3}\left(g_{1} f_{1 k}+x g_{2} f_{2 k}\right) \frac{a b_{j}}{\mu+y \gamma_{j}}-1\right] T_{j}
\end{aligned}
$$

Observe that the inequality (30) is true because $\xi$ and $\Psi$ are positive. In addition by hypothesis (28) and (29) we get $L^{\prime}<0$ for all $\mathrm{j}(=1,2,3)$, therefore the diseases-free equilibrium is globally stable.

Note that if $\frac{a}{H_{3}}<1$ where $H_{3}=\frac{\mu+y \gamma_{3}}{\mu+\gamma_{3}}, i . e$, the reduced infectivity "outweighs" the factor by which mortality is reduced for slow progressors, then (28) implies (29).

Now will do some analysis of this reproductive number for the heterogeneity population. By plotting the reproductive number for the complex model, as a function of $\alpha$ and $\Psi$ and fixing the values for $\omega=1 / 60$, and $\xi=.95$, we obtained the following graph. Which describes the impact of education when the efficacy of education is between 0 and 1 .

As shown in figure 2(parts a, b), $\Re_{0}<1$ as long as $\Psi$ (effectiveness of education) and $\alpha$ (the rate at which susceptibles go to the educated class) are not close to 0 . This means that by educating some small portion of susceptible individuals will have some effect in reducing the generation of secondary newly infective persons, i.e $\Re_{0}$. Hence, education does reduce the reproductive number for the complex model. 

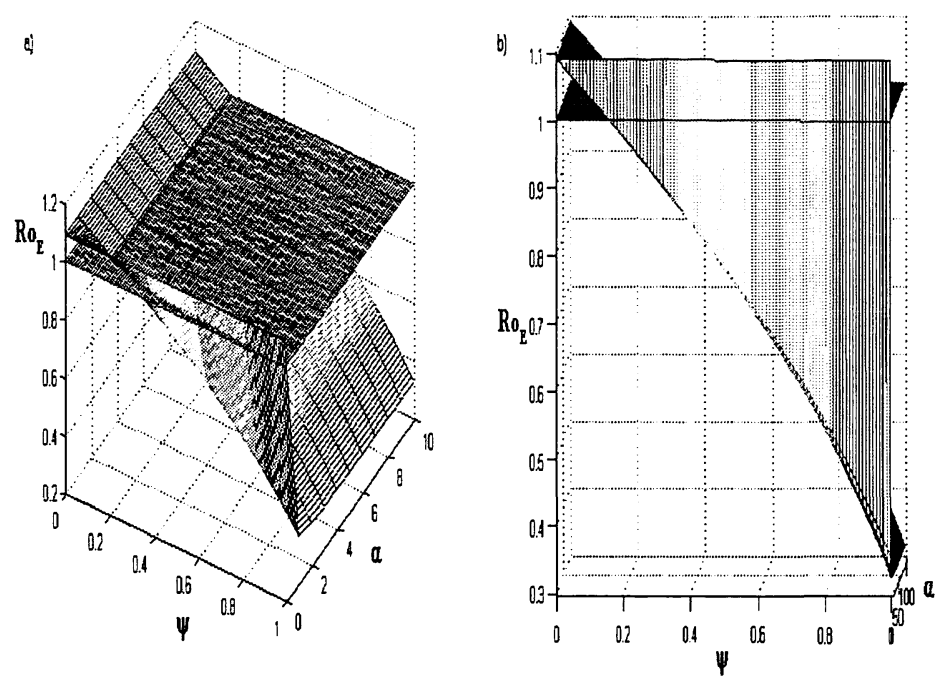

Figure 2: $\Re_{0}$ for education as function of $\Psi \in(0,1]$ and $\alpha \in(0, \infty)$ intercepted with $\Re_{0}=1$ plane.
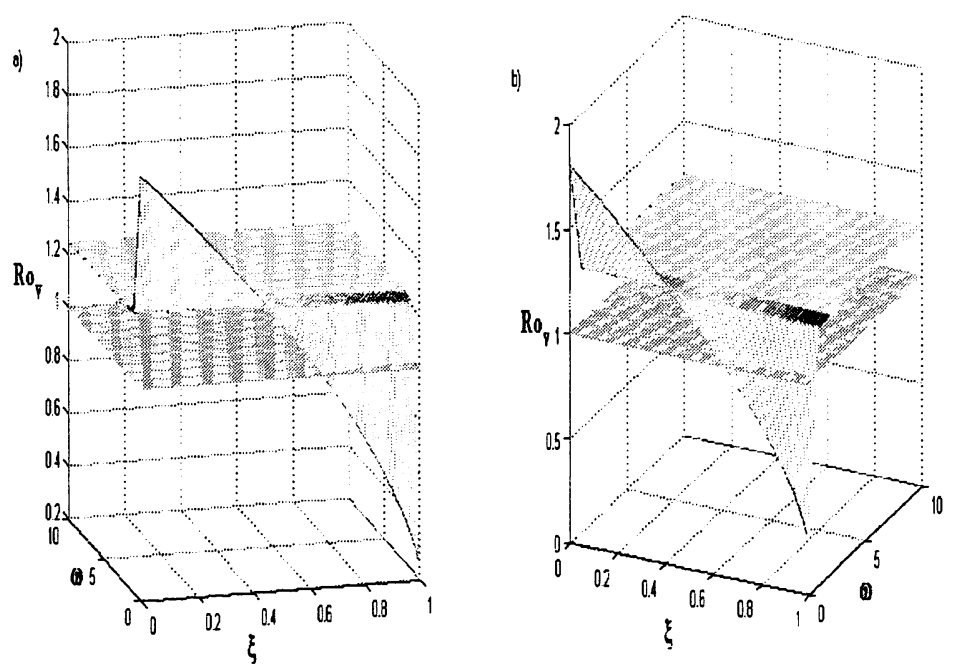

Figure 3: $\Re_{0}$ for vaccine as function of $\xi \in(0,1]$ and $\omega \in(0, \infty)$ intercepted with the plane $\Re_{0}=1$ 
Then to see the impact of vaccination over the reproductive number, we graphed $R e_{0}$ as function of $\xi$ (effectiveness of the vaccine) and $\omega$ (duration protection against HIV) and fixed $\alpha=1 / 32$ and $\xi=0.95$. Figure 3 (parts a, b) shows that $\Re_{0}<1$ if $\omega$ is approximately 0 , and $\xi$ is approaching to one. That is, the vaccine program is effective only when the vaccine is almost perfect and no individuals are losing their immunity to HIV, which implies that they will remain in the vaccinated class. However, this is unrealistic given that no vaccine is perfect, and individuals are only temporarily immune to the virus.

\section{3 $\Re_{0}$ for different cases}

If $m_{i}$ is set to 0 , which implies that no treatment is applied to the population, $\Re_{0}$ will be given by the following relation,

$$
\Re_{0_{I}}=c\left[k_{1}\left(f_{11} \frac{\beta_{1}}{\eta_{1}}+f_{12} \frac{\beta_{2}}{\eta_{2}}+f_{13} \frac{\beta_{3}}{\eta_{3}}\right)+x k_{2}\left(f_{22} \frac{\beta_{2}}{\eta_{2}}+f_{23} \frac{\beta_{3}}{\eta_{3}}\right)\right]
$$

and the only thing that will change when either vaccination and education or both are applied is $k_{i}$.

Let us first consider the most simple case, which is when only three susceptible classes $S_{1}$, $S_{2}$ and $S_{3}$ and the three infected classes $I_{1}, I_{2}$ and $I_{3}$ are taken into consideration. Then, $k_{i}$ will be denoted by

$$
k_{i}=g_{i}
$$

thus, $\Re_{0_{I}}$ is given by the sum of the secondary infections caused by each of the infected classes contained in our model.

Now, if only vaccination is applied to the population, then $k_{i}$ will be given by

$$
k_{i}=g_{i}\left(1-\xi \frac{\mu}{\mu+\omega} P_{i} \epsilon\right)
$$

$\Re_{0}$ will be reduced based on the fact that $k_{i}$ is being multiplied by a factor which is always less than 1. This factor is denoted by reduction term due to the vaccine, and depends on the efficacy of the vaccine and number of people who were vaccinated.

If only education is applied to the population, then $k_{i}$ will take the following form

$$
k_{i}=g_{i}\left(1-\frac{\alpha}{\mu+\alpha} \Psi\right)
$$

if we require that the education is effective in the population level to slow the spread of the disease, then one would expect that $k_{i}<1$. Notice that for any values of $\mu$ and $\alpha, k_{i}$ is always less than 1 , thus, education will reduce $\Re_{0}$.

Now, if we compare the $k_{i}$ 's of vaccination and education respectively to analyze which of the two have the most effect on the population, we get the following relation

$$
\xi \frac{\mu}{\mu+\omega} P_{i} \epsilon<\frac{\alpha}{\mu+\alpha} \Psi
$$


which indicates that it is better to apply education than vaccination. Otherwise, vaccination would be better, however, this is very unlikely to happen since we will be requiring that the efficacy of the vaccine is almost perfect and we know that all vaccines available only provide temporary immunity.

If both vaccination and education are applied to the population, then the value of $k_{i}$ is given by (27). The joint intervention of treatment and vaccination will reduce the basic reproductive number more than if one or the other are applied. Now, if we wanted to determine which of the two has the most effect in the reduction of $\Re_{0}$, we compared their respective $k_{i}^{\prime} s$ and by doing so we get

$$
\xi<\frac{\alpha}{\mu+\alpha} \Psi
$$

this inequality implies that education will have a greater impact than vaccination when both are present in the population, otherwise vaccination will have a greater effect.

\subsection{The $\Re_{0}$ for the SIT, VSIT and SIET Models}

When treatment is applied to the population, the basic reproductive number denoted by $\Re_{0_{T}}$ with $T$ indicating treatment, is

$$
\Re_{0_{T}}=\frac{1}{2}\left(\Re_{0_{I}}+\sqrt{\Re_{0_{I}}^{2}+4 a \overrightarrow{\Re_{I}} \cdot \overrightarrow{\Re_{T}}}\right)
$$

where

$$
\begin{aligned}
\Re_{0_{I}} & =c\left[k_{1}\left(f_{11} \frac{\beta_{1}}{\eta_{1}}+f_{12} \frac{\beta_{2}}{\eta_{2}}+f_{13} \frac{\beta_{3}}{\eta_{3}}\right)+x k_{2}\left(f_{22} \frac{\beta_{2}}{\eta_{2}}+f_{23} \frac{\beta_{3}}{\eta_{3}}\right)\right] \\
\overrightarrow{R_{I}} & =\left(\frac{k_{1} f_{11} \beta_{1}}{m 1},\left(k_{1} f_{12}+x k_{2} F_{22}\right) \frac{\beta_{2}}{\varphi_{2}},\left(k_{1} f_{13}+x k_{2} f_{23}\right) \frac{\beta_{3}}{\varphi_{3}}\right) \\
\overrightarrow{\Re_{T}} & =\left(\frac{m_{1}}{\eta_{1}}, \frac{m_{2}}{\eta_{2}}, \frac{m_{3}}{\eta_{3}}\right)
\end{aligned}
$$

In a similar manner as studied in the previous section, we will present the reproductive number when treatment is present for different cases and again the only thing that will change for each case are the $k_{i}$. Then, an analysis of how treatment affects $\Re_{0_{T}}$ will be given.

If only susceptibles, infecteds and treateds are considered, then $k_{i}$ will be $g_{i}$. Notice that the square root appears in $\Re_{0_{T}}$ when treatment is present due to the two step processes that a treated individual takes before $\mathrm{s} / \mathrm{he}$ can generate another treated.

Now, when vaccination is added to the previous case $k_{i}$ will be given by (33). In this case we see that $\Re_{0_{T}}$ will be reduced by this factor and by the contribution of treatment which will be presented in the next section.

When education is introduced into the system in addition to treatment $k_{i}$, are denoted by (34). $\Re_{0_{T}}$ is reduced by the factor of $k_{i}$ and the contribution of treatment. 
To examine the effectiveness of treatment not only in the individual level, but also in the population level to slow down the spread of the disease we took the partial derivatives of $\Re_{0_{T}}$ with respect to $m_{i}$ and look for conditions under which the derivatives are less than 0 .

For convenience let,

$$
\begin{aligned}
& F_{1}=c k_{1} f_{11} \beta_{1} \\
& F_{2}=c\left(k_{1} f_{12}+x k_{2} f_{22}\right) \beta_{2} \\
& F_{3}=c\left(k_{1} f_{13}+x k_{2} f_{23}\right) \beta_{3}
\end{aligned}
$$

Now, by taking the partial derivative of $\Re_{0_{T}}$ with respect to $m_{1}$ we get

$$
\frac{\partial \Re_{0_{T}}}{\partial m_{1}}=\frac{F_{1}}{\eta_{1}}\left[\left[\left(F_{1}, F_{2}, F_{3}\right) \cdot\left(\frac{1}{\eta_{1}}, \frac{1}{\eta_{2}}, \frac{1}{\eta_{3}}\right)\right]^{\frac{1}{2}}+4 a\left(\frac{F_{1}}{\varphi_{1}} \frac{F_{2}}{\varphi_{2}}, \frac{F_{3}}{\varphi_{3}}\right) \cdot\left(\frac{m_{1}}{\eta_{1}}, \frac{m_{2}}{\eta_{2}}, \frac{m_{3}}{\eta_{3}}\right)\right]
$$

After some simplifications and by setting $\frac{\partial \Re_{0_{T}}}{\partial m_{1}}<0$ we obtain the following,

$$
a\left(\frac{\mu+\gamma_{1}}{\varphi_{1}}\right)^{2}-\frac{\mu+\gamma_{1}}{\varphi_{1}} \vec{F} \cdot\left(\frac{1}{\eta_{1}}, \frac{1}{\eta_{2}}, \frac{1}{\eta_{3}}\right)-\left(\frac{F_{1}}{\varphi_{1}}, \frac{F_{2}}{\varphi_{2}}, \frac{F_{3}}{\varphi_{3}}\right) \cdot\left(\frac{m_{1}}{\eta_{1}}, \frac{m_{2}}{\eta_{2}}, \frac{m_{3}}{\eta_{3}}\right)<0
$$

hence,

$$
\frac{\partial \Re_{0_{T}}}{\partial m_{1}}<0 \Longleftrightarrow a<H_{1} \vec{F}_{1} \cdot\left(\frac{1}{\mu+\gamma_{1}}, \frac{1}{\eta_{2}}\left(1+\frac{m_{2}}{\varphi_{2}} H_{1}\right), \frac{1}{\eta_{3}}\left(1+\frac{m_{3}}{\varphi_{3}} H_{1}\right)\right)
$$

where

$$
H_{j}=\frac{\mu+\gamma_{j} y}{\left(\mu+\gamma_{j}\right)}
$$

Likewise

$$
\begin{aligned}
& \frac{\partial \Re_{0_{T}}}{\partial m_{2}}<0 \Longleftrightarrow a<H_{2} \vec{F}_{1} \cdot\left(\frac{1}{\eta_{1}}\left(1+\frac{m_{1}}{\varphi_{1}} H_{2}\right), \frac{1}{\mu+\gamma_{2}}, \frac{1}{\eta_{3}}\left(1+\frac{m_{3}}{\varphi_{3}} H_{2}\right)\right) \\
& \frac{\partial \Re_{0_{T}}}{\partial m_{3}}<0 \Longleftrightarrow a<H_{3} \vec{F}_{1} \cdot\left(\frac{1}{\eta_{1}}\left(1+\frac{m_{1}}{\varphi_{1}} H_{3}\right), \frac{1}{\eta_{2}}\left(1+\frac{m_{2}}{\varphi_{2}} H_{3}\right), \frac{1}{\mu+\gamma_{3}}\right)
\end{aligned}
$$

note

$$
H_{i}=1-(1-y) \frac{\gamma_{i}}{\mu+\gamma_{i}} \quad \text { so } \quad \gamma_{i}<\gamma_{j} \Longleftrightarrow H_{i}>H_{j}
$$

by assumption of (2) we have $H_{1}<H_{2}<H_{3}$. 
Now define

$$
h_{i j}\left(m_{j}\right)=\frac{1}{\eta_{j}}\left(1+\frac{m_{j}}{\varphi_{j}} H_{i}\right)
$$

note that

$$
h_{i i}=\frac{1}{\mu+\gamma_{i}}
$$

is independent of all $m_{1}, m_{2}, m_{3}$.

Thus for $i \neq j$,

$$
h_{i j}^{\prime}\left(m_{j}\right)=\frac{1}{\eta_{j}^{2}}\left(\frac{H_{i}}{H_{j}}-1\right)>0 \Longleftrightarrow i>j .
$$

hence,

$$
\begin{gathered}
h_{11}^{\prime}=0, h_{12}^{\prime}<0, h_{13}^{\prime}<0, \\
h_{21}^{\prime}>0, h_{22}^{\prime}=0, h_{23}^{\prime}<0, \\
h_{31}^{\prime}>0, h_{32}^{\prime}>0, h_{33}^{\prime}=0
\end{gathered}
$$

also note

$$
\lim _{m_{j} \rightarrow \infty} h_{i j}\left(m_{j}\right)=\frac{H_{i}}{H_{j}} \frac{1}{\mu+\gamma_{j}}
$$

Therefore, if no treatment program is currently in effect, i.e, $m_{1}=m_{2}=m_{3}=0$, then small increase in any or all the $m_{i}$ will reduce $\Re_{0}$, i.e,

$$
\begin{aligned}
& \frac{\partial \Re_{0}}{\partial m_{1}}(0,0,0)<0 \\
& \frac{\partial \Re_{0}}{\partial m_{2}}(0,0,0)<0 \\
& \frac{\partial \Re_{0}}{\partial m_{3}}(0,0,0)<0
\end{aligned}
$$

if the following inequality obtained from (38)-(40) and (41) is satisfied:

$$
a<\min \left(H_{1}, H_{2}, H_{3}\right) \Re_{0}(0)=H_{1} \Re_{0}(0)
$$

If some treatment is already in effect, i.e., $m_{1}+m_{2}+m_{3}>0$ then changes in the $m_{i}$ will reduce $\Re_{0_{T}}$ if and only if the appropriate one(s) of the conditions in (43) hold for the current values of $m_{1}, m_{2}$ and $m_{3}$. 
Now, if we want to ensure that increments in any $m_{i}$ will reduce $\Re_{0_{T}}$ regardless of the current values for $m_{1}, m_{2}, m_{3}$, then we must have

$$
a<\min \left\{\begin{array}{l}
H_{1} \vec{F} \cdot\left(\frac{1}{\mu+\gamma_{1}}, \frac{H_{1}}{H_{2}} \frac{1}{\mu+\gamma_{2}}, \frac{H_{1}}{H_{3}} \frac{1}{\mu+\gamma_{3}}\right), \\
H_{2} \vec{F} \cdot\left(\frac{1}{\mu+\gamma_{1}}, \frac{1}{\mu+\gamma_{2}}, \frac{H_{2}}{H_{3}} \frac{1}{\mu+\gamma_{3}}\right), \\
H_{1} \vec{F} \cdot\left(\frac{1}{\mu+\gamma_{1}}, \frac{H_{1}}{H_{2}} \frac{1}{\mu+\gamma_{2}}, \frac{H_{1}}{H_{3}} \frac{1}{\mu+\gamma_{3}}\right)
\end{array}\right.
$$

A sufficient but not necessary condition to make this happen is

$$
a<\frac{H_{1}^{2}}{H_{3}} \Re_{0}(0)
$$

since $\frac{H_{1}}{H_{3}}$ is the smallest of the fractions above. Basically, the infectivity should be reduced at least as much as the lifespan is prolonged, with respect to $\Re_{0}(0)$.

Based on the previous results we can see that if both vaccination and treatment are applied to the population, the basic reproductive number denoted by $\Re_{0_{T}}$ (35) with $k_{i}$ given by (33) will be reduced if the above inequalities are satisfied. However, reduction of $\Re_{0_{T}}$ does not guarantee extinction of the disease, since both vaccination and treatment are not completely effective and does not give immunity nor eradicate the HIV respectively. Furthermore, if education and treatment are applied to the population, the basic reproductive number denoted by $\Re_{0_{T}}(35)$ and $k_{i}$ given by (34) will be reduced. 


\section{Conclusion}

We have presented a novel model to incorporate genetic heterogeneity into HIV/AIDS epidemiology. The basic reproductive number for this model was derived and the relative contributions from different cases were discussed. Our results support the conclusions of Shu-Fang Hsu Schmitz that some integrated intervention strategies (i.e., vaccination and treatment) are far superior to those based on a single approach. The secondary cases produced by the infectedtreated individuals is reduced the most when vaccination, education and treatment are applied to the population. However, education is the most effective in reducing the $\Re_{0}$ without having to fulfill any conditions and/or restrictions. This is because education is more reliable and it is always better to educate than not to. This is not only supported by our deterministic analysis but by the graphical analysis too. This analysis tell us that if we at least effectively educated some small portion of the suceptible individuals this will reduce the generation of secondary infections. In addition, by deterministic analysis we concluded that vaccination will make a difference only if it takes and gives immunity to the susceptible individuals for a longer period of time. The graphical analysis shows that the vaccine program would be effective only when the vaccine is almost perfect and none of the vaccinated individuals are losing their immunity to HIV. In the case of treatment, ' $a$ ', the reduction factor for infectivity must be really small in order for the treatment to reduce the value of $\Re_{0}$. Treatment will reduce $\Re_{0}$ if the infectivity is reduced at least as much as the lifespan is prolonged. Therefore, the education program is a good option in reducing the number of secondary cases produced by the infected-treated individuals, not only because it is less costly at the individual and population level, but because in the long run people can get a lot more from education than from temporary vaccines and treatment. Nevertheless reduction in $\Re_{0}$ from the three different therapeutic approaches to HIV will not guarantee eradication of HIV, since both vaccines and treatment are not completely effective in reducing the infectivity rate. First, because vaccines are temporary and scientists have not developed an everlasting vaccine in way that would lower the number of HIV infection, and antiviral drugs used in treatment is not $100 \%$ effective because after some time the virus can still replicate even in the presence of these drugs. Likewise, even though education has the most impact in reducing the $\Re_{0}$, effectively educating individuals does not mean these individuals will not get infected. It just means that these individuals will help in reducing the spread of the disease. Thus, the spread of the disease will continue to grow, or at least be control since up until now there is no cure for HIV.

\section{Acknowledgments}

This study was supported by the following institutions and grants: National Science Foundation (NSF Grant DMS-9977919); National Security Agency (NSA Grants MDA 904-00-1-0006); Sloan Foundation: Cornell-Sloan National Pipeline Program in the Mathematical Sciences; and the Office of the Provost of Cornell University. In addition, we would like to thank the faculty at MTBI for their support and interest in our project. We thank Christopher KribsZaleta since we couldn't have possibly conducted this research without him. We also thank 
Carlos Castillo-Chavez for all of his advice, and corrections. We would also like to thank Hsu Schmitz for helping us with the material. 


\section{References}

[1] S.F Hsu Schmitz, Effects of treatment and/or vaccination on HIV transmission in homosexuals with genetic heterogeneity. Mathematical Biosciences :- (1999).

[2] S.F Hsu Schmitz, A mathematical model of HIV transmission in homosexual with genetic heterogeneity. Journal of Threoretical Medicine 2:285-296 (2000).

[3] C. Castillo-Chavez, Z. Feng and W. Huang, On the computation of $\Re_{0}$ and role on global stability. Mathematical Approaches for emerging and reemerging infectious diseases, PartI: An Introduction to Models, Methods and Theory. C.Castillo-chavez, S. Blower, D. Krischner, and A-A Yakubu IMA Volume Springer Verlag, Forthcoming 125:229-250.

[4] R. M. Anderson, S. Gupta, R. M. May, Potential of community-wide chemotherapy of immunotherapy to control the spread of HIV-1. Nature 350:356-359 (1991). .

[5] Dean M, Carrington M, Winkler C, et al. Genetic restriction of HIV-1 infection and progression to AIDS by a deletion allele of the CKR5 structural gene. Science 273:1856-1862 (1996).

[6] Detels R, Liu Z, Hennessey K, et al. Resistance to HIV-1 infection. Multicenter AIDS Cohort Study. J Acquir Immune Defic Syndr Hum Retrovirol 7:1263-1269 (1994).

[7] Diekmann O, Heesterbeek JAP and Metz JAJ. On the definition and the computation of the basic reproduction ratio $\Re_{0}$ in models for infectious diseases in heterogeneous populations. Journal of Mathematical Biology 28:365-382 (1990).

[8] Fowke KR, Nagelkerke NJD, Kimani J, et al. Resistance to HIV-1 infection among persistently seronegative prostitutes in Nairobi, Kenya. Lancet 348:1347-1351 (1996).

[9] Hsu Schmitz S-F. Treatment and vaccination against HIV/AIDS in homosexuals with genetic heterogeneity. Mathematical Biosciences (accepted, to appear).

[10] McLean AR and Blower SM. Imperfect vaccines and herd immunity to HIV. Proc. R. Soc. Lond. B 253:9-13 (1993).

[11] Paxton WA, Martin SR, Tse D, et al. Relative resistance to HIV-1 infection of CD4 lymphocytes from persons who remain uninfected despite multiple high-risk sexual exposure. Nature Medicine 2:412-417 (1996).

[12] Phair JP. Keynote address: variations in the natural history of HIV infection. AIDS RES Hum REtrovir 10:883-885 (1994).

[13] Hartwell L, Hood L, Golberg Ml, et al. Genetics and Society. Genetics and Genes to Genomes. McGraw-Hill higher education: New York, 2000, Chapter 7:242-243

[14] S. Begley. AIDS AT 20, Newsweek June 11, 2001:34-37 
[15] G. Cowley. Can He Find A Cure?, Newsweek June 11, 2001:38-41

[16] Quillent C, Oberlin E, Braun J, et al. HIV-1-resistance phenotype conferred by combination of two separate inherited mutations of CCR5 gene. The Lancet 351:14-18 (1998).

[17] Samson M, Libert F, Doranz BJ, et al. Resistance to HIV-1 infection in Caucasian individuals bearing mutant alleles of the CCR-5 chemokine receptor gene. Nature 382:722-725 (1996).

[18] Sheppard HW, Lang W and Ascher MS. The characterization of non-progressors: longterm HIV-1 infection with stable CD4+ T-cell levels. AIDS 7:1159-1166 (1993).

[19] Smith MW, Dean M, Carrington M, et al. Contrasting genetic influence of CCR2 and CCR5 variants on HIV-1 infection and disease progression. Science 277:959-965 (1997).

[20] Winkler C, Modi W, Smith MW, et al. Genetic restriction of AIDS pathogenesis by an SDF-1 chemokine gene variant. Science 279:389-393 (1998).

[21] Freeman S and Herron JC. A Case for Evolutionary Thinking: Understanding HIV. Evolutionary Analysis. Prentice Hall: 1998, Chapter 1:3-25

[22] Thieme, H.R. and C. Castillo-Chavez (1993) How many infection-age dependent infectivity affect the dynamics of HIV/AIDS?, SIAM J. Appl. Math., 53: 1447-1479.

[23] Castillo-Chavez, C., K. Cooke, W. Huang, and S.A Levin (1989a). The role of long incubation periods in the dynamics of HIV/AIDS. Part 1: Single Populations Models, J. Math Biol., 27:373-98.

[24] Castillo-Chavez, C., K.L Cooke, W. Huang, and S.A Levin (1989b). Results on the dynamics for models for the sexual transmission of the human immunodeficiency virus, $J$. Applied Math. Letters, 2(4):327-331. 